\title{
Islands of (In-)stability in High Temperature Superconductors
}

\author{
B. Biehler, B.-U. Runge, and P. Leiderer \\ Department of Physics, University of Konstanz, Universitätsstr. 10, 78457 Konstanz, Germany \\ E-mail: Bjoern.Biehler@uni-konstanz.de
}

(Received April 13, 2004; revised June 4, 2004)

Dendritic flux penetration is a well known phenomenon in high temperature superconductors. We investigated the conditions under which this instability can be observed in different materials. We found three qualitatively different behaviors: dendrite development above a "critical field" and up to a certain temperature, above a "critical field" with no measurable temperature dependence, or no dendrite development at all.

KEY WORDS: dendrite; stability; flux avalanche; magneto-optic; YBCO; $\mathrm{YNi}_{2} \mathrm{~B}_{2} \mathrm{C} ; \mathrm{BSCCO}$

\section{INTRODUCTION}

The dendritic instability of magnetic flux penetration into superconductors was first observed in $1967^{1}$ in niobium samples. It is a sudden redistribution of magnetic flux in a superconducting sample, which under certain conditions can lead to irreversible damage. ${ }^{2}$ The velocity of the redistribution is in the range of $20-200 \mathrm{~km} / \mathrm{s}^{3}$ This avalanche-like behavior can develop spontaneously or can be triggered by a perturbation in an area where shielding currents are present. ${ }^{4,5}$ We conducted a systematic study under which conditions this form of instability can be observed in three kinds of samples: $\mathrm{YBCO}$ and $\mathrm{YNi}_{2} \mathrm{~B}_{2} \mathrm{C}$ thin films, and $\mathrm{BSCCO}$ single crystals.

\section{EXPERIMENTAL SETUP}

Our setup consists of a home-made polarizing microscope built around a continuous-flow cryostat. The cryostat has optical windows on two sides allowing access to the top and bottom of the sample. The 


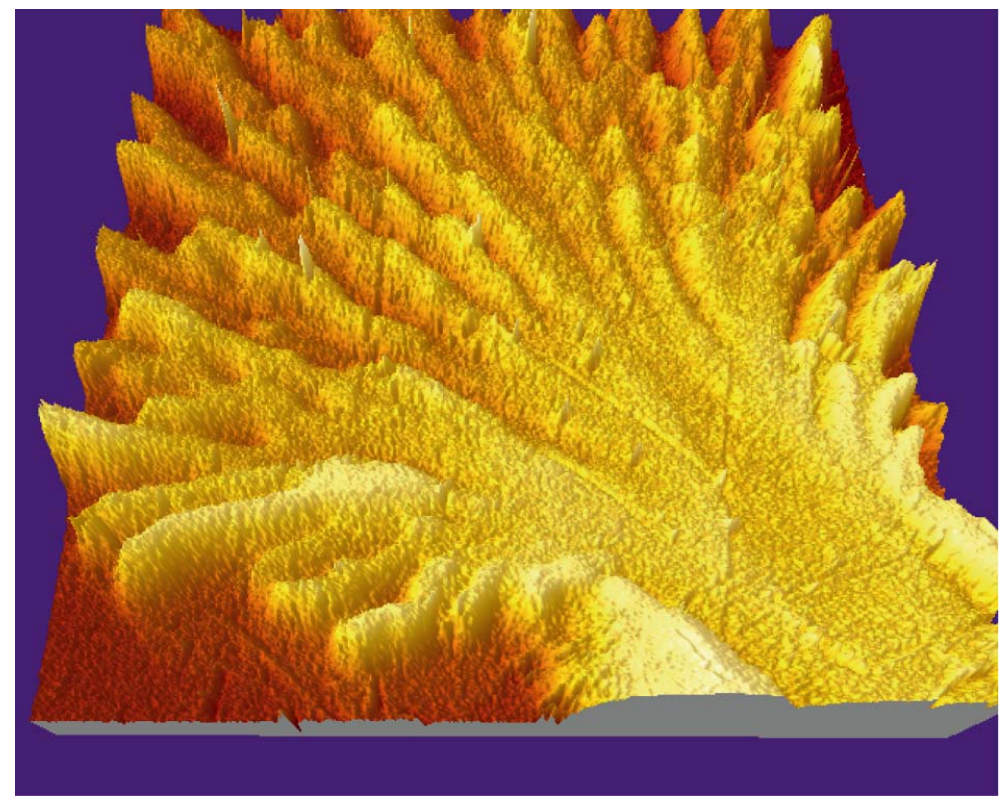

Fig. 1. A pseudo 3D representation of a dendritic flux distribution in $\mathrm{YNi}_{2} \mathrm{~B}_{2} \mathrm{C}$. The figure shows an area of 2.3 by $2.3 \mathrm{~mm}^{2}$.

sample is mounted on a copper plate with a small hole at the sample edge, which is implemented to perturb the superconducting system with a short laser pulse (FWHM $150 \mathrm{fs}$ ). This perturbation is used to trigger the instability we are investigating. On top of the specimen we mounted an iron garnet film with a thickness of some micrometers. The iron garnet shows a pronounced Faraday effect, i.e. the change in polarization is proportional to the magnetic field penetrating the iron garnet. Doing polarization microscopy on this sandwich of sample and iron garnet will lead to an image of the magnetic flux distribution in the superconducting sample, see Fig. 1.

\section{EXPERIMENT AND RESULTS}

A typical experiment was conducted as follows: we cooled the superconductor to the desired temperature in zero field. The external field was then applied by copper coils. This results in a flux pattern governed by shielding currents flowing mainly at the sample edges. No instabilities were observed at this stage. Consecutively, we heated the edge of the superconductor with the focused laser pulse. Here two different 


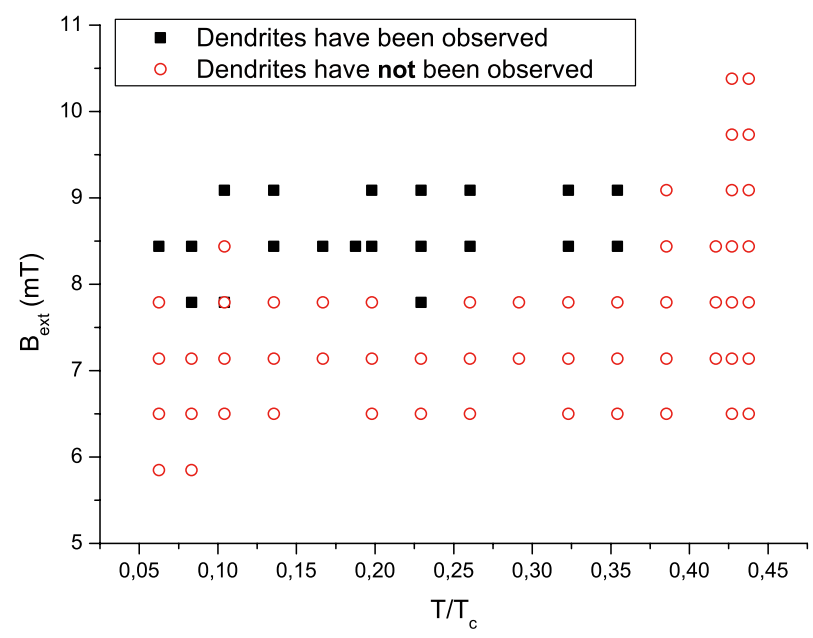

Fig. 2. Phase diagram of an $330 \mathrm{~nm}$ thick YBCO film. Note that no dendrites were observed for $T / T_{c}>0.37$.

kinds of flux redistribution have been observed. Either the flux penetrated homogeneously into the superconductor or formed elongated, branching structures (dendrites) as seen in Fig. 1. Note that a sudden but homogeneous flux penetration would be undistinguishable for us, i.e. jumps in magnetization measurements as in might not be visible as in reference 1. Before the next experiment the superconductor was heated above $T_{\mathrm{c}}$ in zero field to clear the sample of all remanent flux.

\subsection{YBCO}

The YBCO sample was a $330 \mathrm{~nm}$ thick film on a strontium titanate substrate. The instability island in YBCO is shown in Fig. 2. The circles show settings where homogeneous penetration had been observed and the squares indicate the observation of dendrites. One can note that above $37 \%$ of $T_{\mathrm{c}}$ no dendrites can be observed, and that a minimum field of $8 \mathrm{mT}$ is required. A similar behavior was found in $\mathrm{MgB}_{2}$ (not shown here), where both a critical field and a critical temperature have been observed, which separate areas of stability from unstable regions.

\section{2. $\mathrm{YNi}_{2} \mathrm{~B}_{2} \mathrm{C}$}

The $\mathrm{YNi}_{2} \mathrm{~B}_{2} \mathrm{C}$ sample we investigated was a film of $\approx 300 \mathrm{~nm}$ thickness on a $\mathrm{MgO}$ substrate. Also in this material a certain field is needed above which dendrites can be observed. However, contrary to YBCO there 


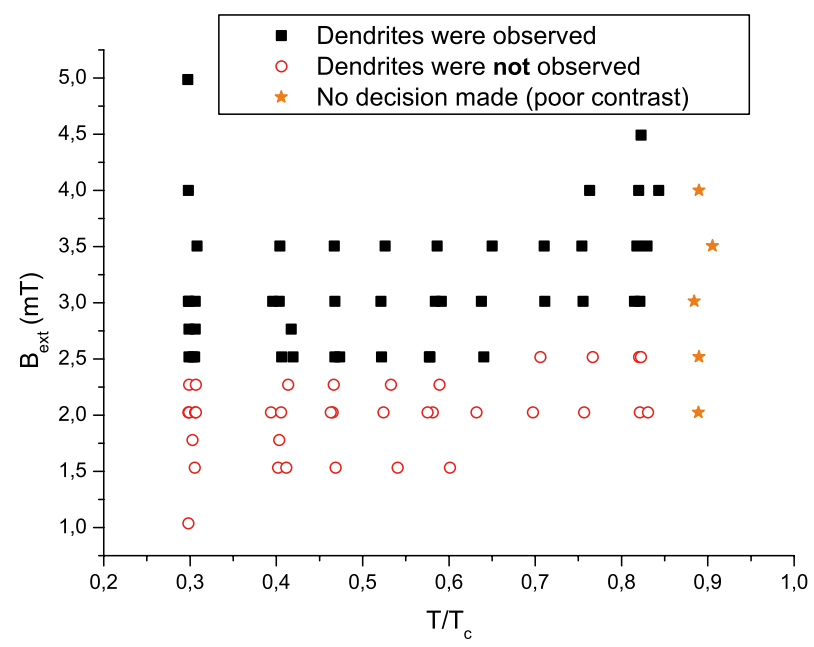

Fig. 3. Phase diagram of a $\mathrm{YNi}_{2} \mathrm{~B}_{2} \mathrm{C}$ film. There is seemingly no upper temperature threshold above which no dendrite development is possible.

is apparently no temperature limit above which dendrite development is hindered. The reason why the measurements do not reach all the way up to $T_{\mathrm{c}}$ is, that the critical currents decrease with increasing temperature. This leads to a deterioration of contrast in the magneto-optic images, which made a discrimination between homogeneous and dendritic penetration impossible in this temperature range (Fig. 3).

\subsection{BSCCO}

BSCCO was in the form of a single crystal. The dimensions were approximately $1 \times 0.4 \mathrm{~mm}^{2}$ with a thickness in the $10 \mu \mathrm{m}$ range. The pinning strength in this sample was lower than in the thin films. Therefore a relaxation in the range of several seconds was observed, which can be understood in terms of a reduced pinning potential or reduced density of pinning centers. As can be seen in Fig. 4, we did not observe any dendrites in this sample.

\section{DISCUSSION AND OUTLOOK}

Most magnetization measurements on superconductors are conducted by slowly varying a quasi DC field. The sweep rate $\dot{B}$ is usually a critical parameter in those measurements, since it is found that a high sweep rate 


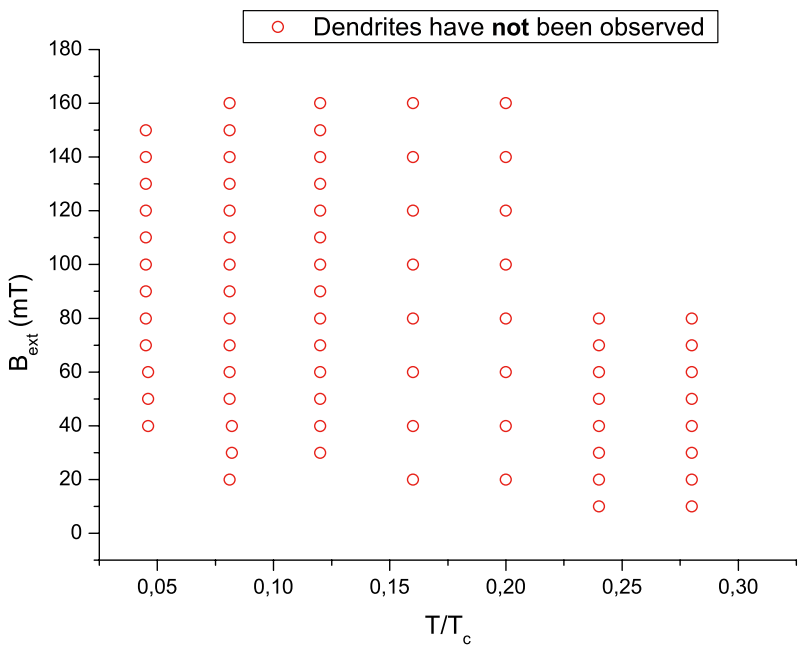

Fig. 4. In BSCCO single crystals, even at high fields and low temperature no dendrites were observed.

leads to a lower instability starting field $B_{\mathrm{fi}} \cdot{ }^{6}$ This is not the case in our setup. Since all relevant time scales are much longer than the perturbation by the laser pulse $\left(\mathcal{T}_{\text {FWHM }}\right)$, it can be viewed as quasi instantaneous, which corresponds to the limiting case of $\dot{B} \rightarrow \infty$.

A stability criterion from Mints and Rakhmanov ${ }^{7}$ was stated in reference 8:

$$
\frac{\mu_{o} J_{c}(T) w^{2}}{c}\left|\frac{\mathrm{d} J_{c}}{\mathrm{~d} T}\right| \equiv \beta(<1 \text { for stability })
$$

$w$ is a typical dimension of the sample and $c$ is the specific heat. For $\beta>$ 1 the flux distribution becomes unstable upon small perturbations. Further it is predicted that the applied field where the first instability can be observed is on the order of

$$
B_{\mathrm{fi}} \approx \sqrt{\mu_{0} c\left(T_{c}-T\right)} .
$$

If we assume that we are able to nucleate dendrites as soon as we reach the stability limit given by Eq. 1, we can compare our results with the theory. The above formula is correct for the adiabatic limit, ${ }^{9}$ which is certainly true for YBCO at low temperatures $(T<10 \mathrm{~K})$. The heat capacity is low $(<1 \mathrm{~J} /(\mathrm{mol} \mathrm{K})$ for $T<13 \mathrm{~K})$, but raises rapidly for higher temperatures to values of $24 \mathrm{~J} /(\mathrm{mol} \mathrm{K})$ for $T=35 \mathrm{~K}^{10}$ For temperatures around 
$30 \mathrm{~K}$ the adiabatic limit might break down, since the thermal conductivity peaks at approximately this value. ${ }^{11}$ These two facts might help to clarify why we see the temperature limit in $\mathrm{YBCO}$ and no such limit in the case of $\mathrm{YNi}_{2} \mathrm{~B}_{2} \mathrm{C}$. Here the heat capacity varies in the examined temperature range only between $\approx 0.1$ and $1.2 \mathrm{~J} /(\mathrm{mol} \mathrm{K}) .{ }^{12}$ This relatively small heat capacity change might not be sufficient to suppress dendrite development.

However, we do not observe the raise in the field where the first instability is expected (Eq. 2): Given the above numbers we would expect in the case of YBCO at least a variation of the field $B_{\mathrm{fi}}$ on the order of the square root of the heat capacity variation, i.e. at least a factor of 4 . This is not observed.

In the case of BSCCO the "heat capacity argument" cannot be used to explain the absence of dendrites. It varies between $0.02 \mathrm{~J} /(\mathrm{mol} \mathrm{K})$ at $T=4 \mathrm{~K}$ to $0.2 \mathrm{~J} /(\mathrm{mol} \mathrm{K})$ at $25 \mathrm{~K}^{13}$ which is much lower than the values for YBCO. If we compare our BSCCO data, with magnetization data obtained in ${ }^{14}$ by Kopelevich et al. we find a discrepancy. We did not observe flux jumps in our sample in the same regime as Kopelevich et al. One has to admit, however, that the jumps reported there are too minute for us to see. On the other hand a striking feature is reported, namely the instability ceases to exist at high fields. This is in disagreement with our data for $\mathrm{YBCO}$, where the instability is only observed at high fields. For $\mathrm{YBCO}$ and $\mathrm{YNi}_{2} \mathrm{~B}_{2} \mathrm{C}$ our result agrees with data of magnetization measurements obtained in Refs. 15 and 16, where no jumps were seen below a certain field.

If the pinning site density/strength is relatively high, the system is unable to relax to a state near the equilibrium, and raising the temperature is not sufficient any more to overcome the instability $\left(\mathrm{YNi}_{2} \mathrm{~B}_{2} \mathrm{C}\right)$. In the case of BSCCO, on the other hand, due to the low pinning potential the system is always able to relax homogeneously. To test this hypothesis we are planning to irradiate a BSCCO sample with highenergy ions to introduce additional pinning centers as was proposed very recently in Ref. 8. This should lead to the development of a dendritic instability.

\section{ACKNOWLEDGMENTS}

We want to thank H. Kinder et al. (TU München, Germany) for the YBCO samples, L. Schultz et al. (IWF Dresden, Germany) for the borocarbide samples, and Y. Yeshurun et al. (Bar-Ilan University, Israel) for the BSCCO single crystals. This work was supported by the GermanIsraeli Foundation. 


\section{REFERENCES}

1. M. R. Wertheimer and J. le G. Gilchrist, J. Phys. Chem. Solids 28, 2509 (1967).

2. P. Brüll, D. Kirchgässner, P. Leiderer, P. Berberich, and H. Kinder, Ann. Physik $1243-$ 247 (1992).

3. U. Bolz, D. Schmidt, B. Biehler, B. -U. Runge, and P. Leiderer, Europhys. Lett. 64 (4) 517-523 (2003).

4. P. Leiderer, J. Boneberg, P. Brüll, V. Bujok, and S. Herminghaus, Phys. Rev. Lett 71 (16) 2646 (1993) .

5. A. Bobyl, D. Shantsev, T. Johansen, W. Kang, H. Kim, E. Choi, and S. Lee, Appl. Phys. Lett. 80 (24) 4588-4590 (2002).

6. S. Khene and B. Barbara, Solid State Comm. 109 727-731 (1999).

7. R. G. Mints and A. L. Rakhmanov, Rev. Mod. Phys. 53 (3) 551-592 (1981).

8. E. Altshuler and T. H. Johansen, Rev. Mod. Phys. 76 (2) 471-487 (2002).

9. R. G. Mints and E. H. Brandt, Phys. Rev. B 54 (17) 12421-12426 (1996).

10. V. G. Bessergenev, Yu. A. Kovalevskaya, V. N. Naumov, and G. I. Frolova, Physica $C$ 245 36-40 (1995).

11. R. K. Williams, J. O. Scarbrough, J. M. Schmitz, and J. R. Thompson, Phys. Rev. B 57 (17) $10923-10928$ (1998).

12. C. C. Hoellwarth, P. Klavins and R. N. Shelton, Phys. Rev. B 53 (5) 2579-2582 (1996).

13. A. Junod, K. -Q. Wang, T. Tsukamoto, G. Triscone, B. Revaz, E. Walker, and J. Müller, Physica C 229 209-230 (1994).

14. Y. Kopelevich and P. Esquinazi, J. Low Temp. Phys. 113 (1/2) 1-9 (1998).

15. N. Kobayashi, T. Nishizaki, Y. Onodera, H. Asaoka, and H. Takei, Chin. J. Phys. 34 (2II) 514-521 (1996).

16. S. C. Wimbush, Mixed State Properties of Rare Earth Nickel Borocarbide Thin Films: Anisotropy and Flux Instability, Ph.D. thesis IFW Dresden (2004). 